\title{
Sliding-Mode-Based Direct Adaptive Fuzzy Controller Design for a Class of Uncertain Multivariable Nonlinear Systems
}

\author{
Wei-Song Lin and Chun-Sheng Chen \\ Department of Electrical Engineering, National Taiwan University, \\ No. 1, Sec. 4, Roosevelt Road, Taipei, Taiwan, R.O.C.
}

\begin{abstract}
A practical design of fuzzy logic controller is presented in which the sliding mode control method and the adaptive control scheme are properly incorporated to enhance robustness and sliding performance in a class of uncertain MIMO nonlinear systems. The proposed fuzzy controller requires no knowledge of an MIMO nonlinear system and facilitates robust properties by fine-tuning the consequent membership functions. By employing the fuzzy descriptions to overcome the interaction among the subsystem, a fuzzy sliding mode controller is used to approximate the equivalent control in the neighborhood of the switching hyperplane. The hitting control is appended to assure that the fuzzy sliding mode control can achieve a stable closed-loop system through Lyapunov stability theory for the trajectories tracking control of a plant with unknown nonlinear dynamics. Finally, the simulation results of a two-link robotic manipulator confirm that the effect of both the fuzzy approximation error and external disturbance on the tracking error can be attenuated efficiently by the proposed method.
\end{abstract}

\section{Introduction}

Conventional control theory is well suited for application where the control efforts can be generated based on analytical model [1-2]. There are inevitable unmodelled nonlinearities and uncertain disturbance in their constructed model where conventional control strategies cannot be easily derived. The latest studies consider adding some computationally intelligent methods to the sliding mode control (SMC) by automatically tuning the control parameters. Particularly, integrating fuzzy set theory and SMC into fuzzy controller design have acquired superior performance [3-8]. This approach retains the positive property of SMC but alleviates the chattering, and the fuzzy control rules can be determined systematically by the reaching condition of the SMC.

This paper will address the problem of controlling an unknown multi-input multi-output (MIMO) nonlinear affined system. The goal is to develop a direct adaptive MIMO fuzzy controller to overcome the interaction among the subsystems by a decoupling neural network and to facilitate robust properties by fine-tuning the consequent membership functions. Firstly, a sliding mode controller for robust tracking control of multivariable nonlinear systems is - developed by assuming that imposed uncertainties are bounded and satisfy matching conditions. The fuzzy logic control is then designed on the basis of the SMC law. A fuzzy sliding mode control (FSMC) is used to approximate the equivalent control in the neighborhood of the switching hyperplane with on-line fuzzy self-tuning parameters subject to parameter variations in the control object. Secondly, the hitting control is appended to assure that the proposed FSMC can result in a closed-loop system that is stable for the trajectories tracking control of a plant with unknown nonlinear dynamics. As a result, we simultaneously guarantee the global stability of the closedloop system and obtain a suitable equivalent control when the nominal mathematic model is unknown in advance. The simulations using the proposed method by a two-link manipulator subject to uncertainties is performed to demonstrate the properties of the developed FSMC.

\section{Problem Formulation}

Consider an MIMO nonlinear system govern by

$$
y^{(r)}=f(x)+G(x) u+d(x, t)
$$

where $y=\left(y_{1}, \cdots, y_{m}\right)^{T}$ and $\boldsymbol{y}^{(r)} \equiv\left(y_{1}^{\left(r_{1}\right)}, \cdots, y_{m}^{\left(r_{m}\right)}\right)^{T}$ denote the output vector and its derivative, respectively, $\boldsymbol{r}=\left(r_{1}, \cdots, r_{m}\right)$ with $\sum_{j=1}^{m} r_{i}=n$ is defined as the system relative degree, $\boldsymbol{u}=\left(u_{1}, \cdots, u_{m}\right)^{r}$ is the input, $\boldsymbol{x}=\left(x_{1}, x_{2}, \cdots, x_{n}\right)^{T}=\left(y_{1}, \cdots, y_{1}^{(n-1)}, y_{2}, \cdots, y_{m}^{\left(r_{m}-1\right)}\right)^{T}$ is the state vector, $f(x)=\left(f_{1}(x), \cdots, f_{m}(x)\right)^{T}, \quad G(x)=$ $\left[\boldsymbol{g}_{1}(x), \cdots, \boldsymbol{g}_{m}(x)\right]^{T}, \quad \boldsymbol{g}_{i}(\boldsymbol{x})=\left(g_{i 1}(x), \cdots, g_{i m}(x)\right)^{T}$ with $g_{i j}>0$ and $f_{i}(x), i=1, \cdots, m$, are unknown functions. and $\boldsymbol{d}(x, t)=\left(d_{1}(x, t), \cdots, d_{m}(x, t)\right)^{r}$ is the disturbance with the properties of standard smoothness and it is assumed to have upper bound $D=\operatorname{Diag}\left[D_{i}\right]$, that is, $d_{i}(x, t) \leq D_{i}, i=1, \cdots, m$.

Let $y_{d}=\left(y_{d 1}, y_{d 2}, \cdots, y_{d m}\right)^{r}$ represents the known desired trajectory, the control aim is to determine a controller for the composite nonlinear system described by (1) so that the tracking error represented by

$$
\underline{e}=\left[e_{1}, \cdots, e_{m}\right]^{T}
$$

with $e_{i}=\left(e_{i}, \dot{e}_{i}, \cdots, e_{i}^{\left(r_{i}-1\right)}\right)^{T}=\left(y_{d i}-y_{i}, \cdots, y_{d i}^{\left(r_{i}-1\right)}-y_{i}^{\left(r_{i}-1\right)}\right)^{T}$, $i=1, \cdots, m$, will be attenuated to an arbitrarily small residual tracking error set. Define a generalized error vector to represent a switching manifold as follows:

$$
s=\left(\begin{array}{c}
e_{1}^{\left(r_{2}-1\right)}+\alpha_{11} e_{1}^{(n-2)}+\cdots+\alpha_{1 \cdot\left(r_{1}-1\right)} e_{1} \\
e_{2}^{\left(r_{2}-1\right)}+\alpha_{21} e_{2}^{\left(r_{2}-2\right)}+\cdots+\alpha_{2 \cdot\left(r_{2}-1\right)} e_{2} \\
\vdots \\
e_{m}^{\left(r_{m}-1\right)}+\alpha_{m 1} e_{m}^{\left(r_{m}-2\right)}+\cdots+\alpha_{m\left(r_{m}-1\right)} e_{m}
\end{array}\right)
$$


and $\Lambda_{1}=\left(\alpha_{11}, \cdots, \alpha_{i r}\right)^{\gamma} \subseteq R^{r_{n}}$ be such that all roots of the polynomial

$$
h_{i}(p)=p^{\left.i r_{1}\right)}-\alpha_{1,} p^{\left(r_{r}-1,\right.}+\cdots+\alpha_{i,(r,-1)} \dot{p}+\alpha_{t r}
$$

are in the open left-half plane, $i=1, \cdots, m$. The aim of sliding mode control law is to force the system states approach the sliding surface and then move along the sliding surface to the origin. This implies that the system dynamics will track reference trajectory asymptotically.

\section{Description of the Fuzzy Logic System}

Various fuzzy models and their control have been successfully applied in many fields [9-12]. The basic configuration of the fuzty logic system comprises four principal components: fuzzifier, fuzzy rule base, fuzzy inference engine and defuzzifier [13]. The fuzzy control rules are the principal factor to determine the performance of a fuzzy controller. The fuzzy system can uniformly approximate nonlinear continuous functions to arbitrary accuracy [14-15]. Thus we will introduce fuzzy systems, which are expressed as a series expansion of fuzzy basis functions

The fuzzy logic system performs a mapping from $U=R^{n} \quad$ to $\quad V=R^{m}$. Let $U=U_{1} \times \cdots \times U_{n}^{\prime} \quad$ and $V=V_{1} \times \cdots \times V_{m}$ where $U_{k} \subset R, k=1,2, \cdots, n$ and $V, \subset R$, $i=1,2, \cdots, m$. A multivariable system can be controlled by the following $N$ linguistic rules

$$
\begin{aligned}
& R^{\prime \prime \prime}: \text { IF } x_{1} \text { is } A_{1}^{\prime} \text { and } \cdots \text { and } x_{n} \text { is } A_{n}^{\prime} \\
& \text { THEN } z_{1} \text { is } B_{1}^{\prime} \text { and } \cdots \text { and } z_{m} \text { is } B_{m}^{\prime}
\end{aligned}
$$

where $l=1, \cdots, N, x_{k}, k=1,2, \cdots, n$, are the input variables to the fuzzy system, $z_{1}, i=1,2, \cdots, m$, are the output variables of fuzzy system, and the antecedent fuzzy sets $A_{k}^{\prime}$ in $U_{k}$ and the consequent fuzzy sets $B_{i}^{\prime}$ in $V_{l}$ are linguistic terms characterized by the fuzzy membership functions $\mu_{A_{i}^{\prime}}\left(x_{k}\right)$ and $\mu_{B_{i}^{\prime}}\left(z_{i}\right)$, respectively. The fuzzy logic system with center-average defuzzifier, product inference and singlcton fuzzifier is defined as [14]

$$
z_{1}(x)=\frac{\sum_{l=1}^{N} \mu^{\prime}(x) \cdot q_{l}^{\prime}}{\sum_{l-1}^{N} \mu^{\prime}(x)}
$$

where $\mu^{\prime}(x)=\Pi_{k-1}^{n} \mu_{A_{k}^{\prime}}\left(x_{k}\right)$ is the matching degree of the lth rule, and $q_{1}^{\prime}$ is the center of the consequent membership function of the th rule. If $q_{1}^{\prime}$ is chosen as the design parameter, the adaptive fuzzy system can be viewed as the type of neural network [16]. Therefore, (6) can be rewritten as

$$
z_{i}(x)=\phi_{,}^{\top} \psi(x)
$$

where $\phi_{i}=\left(q_{1}^{i}, \cdots, q_{1}^{\prime \prime}\right)^{\prime}$ is a parameter vector, and $\psi(x)=\left(\xi_{1}, \cdots, \xi_{, N}\right)^{T}$ is a regressor, and where the fuzzy basis function is defined as [14]

$$
\xi_{i}=\frac{\Pi_{k=1}^{n} \mu_{j}\left(x_{i}\right)}{\sum_{n=1}^{n}\left(\Pi_{k=1}^{n} \mu_{y}\left(x_{k}\right)\right)}
$$

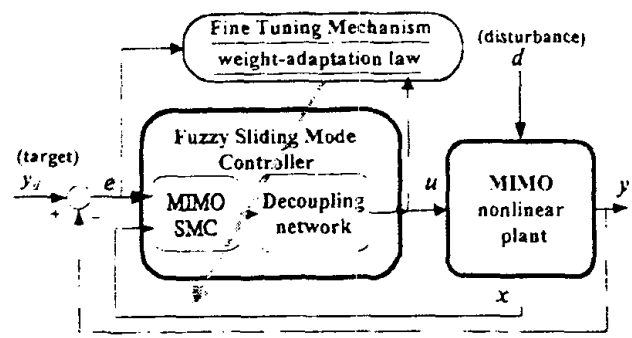

Fig. 1 Configuration of the adaptive FSMC system.

\section{Adaptive Fuzzy Sliding Mode Controller}

The proposed adaptive fuzzy sliding mode controller is composed of the following three parts: an MIMO SMC, a fine-tuning mechanism on the consequent membership functions of the multi-layer fuzzy system, and a decoupling network shown in Fig. 1 [17]. The multi-layer fuzzy system and the decoupling network are nominal designs based on on-line approximation of the unknown nonlinear functions of the plant. The fine-tuning mechanism is designed to encounter the equivalent uncertainty resulted by the plant uncertainty, the function approximation error, or the external disturbances.

Let $u_{i}^{0}$ be the output of the system's ith MIMO SMC. Then, for the system given in (1), the $i$ th sliding surface is $s_{t}$. Hence, this MIMO SMC also has $m$ sliding surfaces to form a switching manifold so that the system exhibits desirable behavior when its trajectories are confined in the sliding surfaces. If the control law is designed such that the sliding mode exists on $s_{i}=0$, $i=1, \cdots, m$, the system error dynamics is dictated by the linear dynamic equation (3). Since (3) satisfies Hurwitz stability criterion from (4), maintaining system states on sliding surface $s$ for all $t>0$ is equivalent to the tracking problem $y=y_{d}$, that is, it is required that the system errors converge to zero. Thus, the tracking control problem can be formulated by keeping the error vector in (2) on the sliding surface defined as follows:

$$
\dot{\boldsymbol{s}}=\boldsymbol{y}_{d}^{(r)}-f(\boldsymbol{x})-\boldsymbol{G}(\boldsymbol{x}) \boldsymbol{u}-\boldsymbol{d}(\boldsymbol{x}, t)+\left(\begin{array}{c}
\sum_{i=1}^{r_{i}-1} \alpha_{1 i} e_{1}^{\left(r_{1}-i\right)} \\
\sum_{t=1}^{r_{2}-1} \alpha_{2 i} e_{2}^{\left(r_{2}-i\right)} \\
\vdots \\
\sum_{t=!}^{r_{m}-1} \alpha_{m i} e_{m}^{\left(r_{m}-i\right)}
\end{array}\right)
$$

In the design of sliding mode controller, an equivalent control is given first such that each state Lyapunov-like condition holds for system stability [18]:

$$
\frac{1}{2} \frac{d}{d t}\left(s_{i}^{2}\right) \leq-\eta_{i} \mid s, \eta_{1}>0, i=1, \cdots, m
$$

or in sum: 


$$
\frac{1}{2} \frac{d}{d t}\left(s^{2} s\right) \leq-\sum_{i=1}^{m} \eta_{i}\left|s_{i}\right|, \eta_{i}>0, i=1, \cdots, m
$$

Inequality (10) constrains trajectories to point towards the surface $s_{i}(t)$ such that the distance to sliding surface decreases along all system trajectories, and is referred to as the reaching condition. That is, the states of the system are driven from any initial state to the eventual sliding surface on which sliding mode control takes place.

If the function $f, G$ and $d$ of nonlinear MIMO systems (1) are known and does not take the interconnections among subsystems into consideration, then the control law $\boldsymbol{u}^{\circ}$ can be chosen as follows

$$
\boldsymbol{u}^{0}=\hat{\boldsymbol{P}}^{-1}\left(\left(\begin{array}{c}
\sum_{i=1}^{r_{1}-1} \alpha_{1 i} e_{1}^{\left(r_{1}-1\right)} \\
\sum_{i=1}^{r_{2}-1} \alpha_{2 i} e_{2}^{\left(r_{2}-i\right)} \\
\vdots \\
\sum_{i=1}^{r_{m}-1} \alpha_{m i} e_{m}^{\left(r_{m}-1\right)}
\end{array}\right)-f-d+y_{d}^{(r)}+K \operatorname{sgn}(s)\right)
$$

where $\hat{\boldsymbol{P}}=\operatorname{Diag}\left[g_{i j}\right], \quad K=\operatorname{Diag}\left[K_{i}\right]$ is $m \times m$ positive definite diagonal gain matrix with $K_{i}>0$, $\operatorname{sgn}(s) \equiv\left(\operatorname{sgn}\left(s_{1}\right), \cdots, \operatorname{sgn}\left(s_{m}\right)\right)^{T}$ and $\operatorname{sgn}\left(s_{i}\right)$ is defined as

$$
\operatorname{sgn}\left(s_{i}\right)=\left\{\begin{array}{cc}
1 & s_{i}>0 \\
0 & s_{i}=0, \\
-1 & s_{i}<0
\end{array} \quad i=1, \cdots, m .\right.
$$

Therefore the optimal control is

$u_{i}^{*}=g_{i i}^{-1}\left[\sum_{j=1}^{r_{i}-1} \alpha_{i j} e_{i}^{\left(r_{i}-j\right)}-f_{i}-d_{i}+y_{d i}^{\left(r_{i}\right)}+h_{i} \operatorname{sgn}\left(s_{i}\right) \cdot \eta_{i}\right]$

where $\eta_{i}>0, h_{i}=\left\{\begin{array}{ll}1 & \text {, if } s_{i} \neq 0 \\ 0 & \text {, if } s_{i}=0\end{array}\right.$.

This optimal sliding mode control input $u_{i}^{*}$ guarantees the reaching condition of $(10)$.

Since the control of MIMO nonlinear systems directly use the sliding mode control but does not take the interconnections among subsystems into consideration, the interconnections compensating network is needed. Thus the proposed sliding mode controller has a neural part to release the interaction among the subsystems. The output of the controller is combined with $\boldsymbol{u}^{0}$ and its modification by decoupling network

$$
u(t)=u^{0}(t)+M u^{0}(t)
$$

To derive a stable weight adaptation in control matrix, the matrix $M$ be chosen as

$$
\boldsymbol{M}=-\left(\boldsymbol{I}_{m}+\hat{\boldsymbol{C}}^{-1} \hat{\boldsymbol{P}}\right)^{-1}
$$

where $I_{\mathrm{m}}$ denotes a $m \times m$ identity matrix and

$$
\hat{C}=\left[\begin{array}{cccc}
0 & g_{12} & \cdots & g_{1 m} \\
g_{21} & 0 & \cdots & g_{2 m} \\
\vdots & \vdots & \ddots & \vdots \\
g_{m)} & g_{m 2} & \cdots & 0
\end{array}\right]
$$

Using (12), (13), (14), (15) and the matrix inversion $(A+B C D)^{-1}=A^{-1}-A^{-1} B\left(D A^{-1} B+C^{-1}\right)^{-1} D A^{-1}$ [19], the formulation of MIMO SMC resolves into

$$
u^{*}=G^{-1}\left(\left[\begin{array}{c}
\sum_{i=1}^{r_{1}-1} \alpha_{1 i} e_{1}^{\left(r_{j}-i\right)} \\
\sum_{i=1}^{r_{2}-1} \alpha_{2 i} e_{2}^{\left(r_{2}-i\right)} \\
\vdots \\
\sum_{i=1}^{r_{m}-1} \alpha_{m i} e_{m}^{\left(r_{m}-i\right)}
\end{array}\right]-f-d+y_{d}^{(r)}+K \operatorname{sgn}(s)\right)
$$

where $\boldsymbol{G}=\hat{\boldsymbol{C}}+\hat{\boldsymbol{P}}$. By plugging $\boldsymbol{u}^{*}$ into (9), we will have $\dot{s}=-K \operatorname{sgn}(s)$. Thus, the reaching condition (10) can be easily verified.

In this paper we use direct adaptive fuzzy controller (DAFC), therefore, the parameters of the controller are directly adjusted to reduce some norm of the output error between the plant and the reference model. Due to the existing of fuzzy approximation errors and external disturbances, simply an equivalent control term cannot ensure the stability of the closed-loop system, it is necessary to preserve a hitting control to deal with them. Suppose that the control $\boldsymbol{u}$ due to the DAFC is the summation of a basic fuzzy logic system $\hat{\boldsymbol{u}}(\boldsymbol{x} \mid \phi)$ and a

$$
\begin{aligned}
\text { hitting control } \hat{u}_{h}\left(\hat{\boldsymbol{u}}_{h}=G^{-1} u_{h}\right) \\
\qquad \boldsymbol{u}=\hat{\boldsymbol{u}}(x \mid \underline{\phi})+\hat{u}_{h}
\end{aligned}
$$

where $\quad \phi=\left[\varphi_{1}, \cdots, \varphi_{m}\right] \quad, \quad \hat{u}=\left(\hat{u}_{1}, \cdots, \hat{u}_{m}\right)^{T} \quad$ with $\hat{u}_{i}\left(x \mid \varphi_{i}\right)=\varphi_{i}^{I} \cdot \xi_{u}(x)$, where $\xi_{n}(x)=\left(\xi_{u 1}, \cdots, \xi_{u N}\right)^{r}$ is a vector of fuzzy bases, $\varphi_{i}=\left(\phi_{i 1}, \cdots, \phi_{i N}\right)$ is the corresponding parameters of fuzzy logic systems, $i=1, \cdots, m$.

\section{Learning Algorithm and Performance Analysis}

In direct adaptive fuzzy control, linguistic fuzzy control rules can be directly incorporated into the controllers and the parameters of the controller are directly adjusted to reduce some norm of the output error between the plant and the reference model. As far as the adaptation of the controller parameters are concerned the input applied to one subsystem affecting the other subsystem. Our approach to the solution of such a problem is based on to derive the proper direct adaptive fuzzy control law for the plant model whose structure is represented by exploiting the advantages of the DAFC and the IAFC (indirect adaptive fuzzy controller) into a single controller i.e. both the fuzzy control nules and the fuzzy descriptions can be incorporated into a single controller. Thus the unknown functions $G(x)$ is estimated and the controller is chosen by assuming the estimated parameters being able to representing the true of the plant parameters. This is similar that the IAFSMC (indirect adaptive fuzzy sliding mode control) uses the fuzzy system as approximator for the dynamic systems [17]. In this section, we firstly show how to derive an adaptive law to adjust the controller parameters such that the DAFC can optimally approximate the equivalent control of the FSMC under the situation of unknown functions $f$ and $G$. Then, we construct the hitting control to guarantee system's stability by the Lyapunov 
theory so that the ultimately bounded tracking is accomplished.

We now adopt the control $\boldsymbol{u}=\hat{\boldsymbol{u}}(x \mid \phi)+\hat{\boldsymbol{u}}_{\boldsymbol{h}}$ as (17) where the hitting control $\hat{\boldsymbol{u}}_{h}=\boldsymbol{G}^{-1} \boldsymbol{u}_{h}$, and the fuzzy logic system $\hat{u}_{\imath}\left(x \mid \varphi_{i}\right)$ as $(7)$ is

$$
\dot{u}_{i}\left(x \mid \phi_{i}\right)=\phi_{i}^{T} \cdot \xi_{n}(x)=\xi{ }^{T}(x) \cdot \phi_{i}
$$

where $\xi_{n}(x)$ is vector of fuzzy bases, $\phi$, is the corresponding parameters of fuzzy logic systems, $i-1, \cdots, m$. Define the parameters $\varphi_{i}^{*} \in R^{N}$ of the best function approximation as

$$
\varphi_{i}^{*} \equiv \arg \min _{\phi_{i} \in \Omega_{\phi_{i}}}\left[\sup _{x \in \Omega_{i},}\left|u_{i}(x)-\hat{u}_{i}\left(x \mid \varphi_{i}\right)\right|\right]
$$

where $\Omega_{\phi}$ is constraint sets for $\varphi_{i}, i=1, \cdots, m$, defined as $\Omega_{\phi_{1}}=\left\{\varphi_{1} ;\left|\varphi_{l}\right| \leq M_{\phi_{1 \text {. Har }}}\right\}$ where $M_{\phi_{\text {i.tAr }}}$ are specified by the designer. $\Lambda$ fter some straightforward manipulations, the sliding surface equation (9) with the fuzzy control law $u$ in (17) to replace $u^{*}$ in (16) can be rewritten as

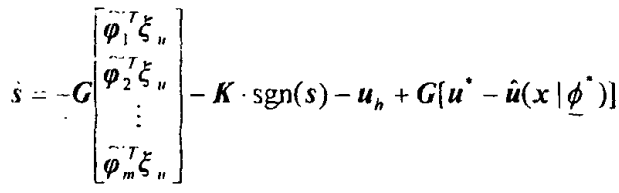

where $\ddot{\varphi}_{i}=\varphi_{i}-\varphi_{i}$ denotes the parameter estimation errors with $\phi=\left\lfloor\varphi_{1}, \cdots, \varphi_{m}\right\rfloor$. Our design objective involves specifying the control and adaptive laws for $\varphi$, such that the reaching condition (10) is guaranteed.

Theorem I: Consider nonlinear plant (1) with controller (17), the tracking error allows to use the following adaptive law and hitting control as

$$
\begin{aligned}
& \dot{\varphi}_{1}=\rho, s^{\top} g_{i} \xi_{n} \\
& u_{t, t}=\operatorname{sgn}\left(s_{,}\right)\left[\left.\left|f_{1} !_{\max }+\sum_{j=1}^{m}\right| g_{i j}\right|_{\max } \cdot\left|\hat{u}_{j}\right|+\left|y_{d i}^{\left(r_{t}\right)}\right|\right. \\
& \left.+\left|\sum_{i=1}^{r_{j}-1} \alpha_{i j} e_{i}^{\left(r_{i}-1\right)}\right|+D_{1}\right]
\end{aligned}
$$

where $i=1, \cdots, m$. After straightforward manipulation, the time derivative of $V$ is obtained as $\dot{V}=\boldsymbol{s}^{r} \dot{\boldsymbol{s}} \leq \mathbf{0}$.

Proof: Consider the Lyapunov candidate

$$
V=V_{1}+V_{2}+\cdots+V_{m}=\frac{1}{2}\left(s^{T} s+\sum_{i=1}^{m} \frac{1}{\rho_{i}} \tilde{\varphi}_{i}^{T} \tilde{\varphi}_{i}\right)
$$

where $V_{1}=\frac{1}{2}\left(s_{1}^{T} s_{t}+\frac{1}{\rho} \tilde{\varphi}_{i}^{T} \tilde{\varphi}_{l}\right), i=1, \cdots, m$. By the fact $\dot{\varphi}_{1}=\dot{\varphi}_{i}$ and (19), we obtain the derivative of $V$ as

$$
\dot{V}=\dot{V}_{1}+\dot{V}_{2}+\cdots+\dot{V}_{m}
$$

where

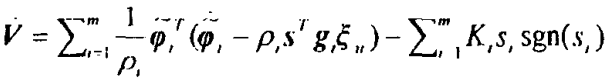

$$
\begin{aligned}
& -\sum_{r=1}^{m} s_{1} u_{h}+s^{\prime} G\left[u^{*}-\hat{u}\left(x \mid \phi^{*}\right)\right]
\end{aligned}
$$

where $g_{1}(x)=\left(g_{i 1}(x), \cdots, g_{1 m}(x)\right)^{T}, i=1, \cdots, m$. If we choose the adaptive law as $\dot{\bar{\varphi}}_{1}=\rho_{c} s^{r} g_{1} \check{\xi}_{\text {, }}$ and the optimal control (16), then

$$
\begin{aligned}
\dot{V}_{i}= & -K_{i} s_{i} \operatorname{sgn}\left(s_{i}\right)+s_{i}\left[\sum_{j=1}^{m} g_{i j} u_{j}-\sum_{j=1}^{m} g_{i j} \hat{u}_{j}\right]-s_{i} u_{h i} \\
= & -K_{i} s_{i} \operatorname{sgn}\left(s_{i}\right)+s_{i}\left[\sum_{j=1}^{r_{j}-1} a_{i j} e_{i}^{\left(r_{i}-i\right)}-f_{i}-d_{i}+y_{d i}^{\left(r_{i}\right)}\right. \\
& \left.+K_{i} \operatorname{sgn}\left(s_{i}\right)-\sum_{j=1}^{m} g_{i j} \hat{u}_{j}\right]-s_{i} u_{h \prime} \\
\leq & \mid s_{i}\left[\left|\sum_{j=1}^{r_{j}} \alpha_{i j} e_{i}^{\left(r_{i}-i\right)}\right|+\left|f_{i}\right|+D_{i}+\left|y_{d i}^{\left(r_{i}\right)}\right|+\sum_{j=1}^{m}\left|g_{i j} \hat{u}_{i}\right|\right] \\
& -s_{i} u_{h r}
\end{aligned}
$$

We use the fact that $u_{h i}$ has the same sign with $s_{i}$, the $u_{h i}$ can be implemented in (21) such that $\dot{V}_{i} \leq 0, i=1, \cdots, m$.

In order to complete the FSMC design, it is necessary to show that the hitting control is enough to force the state trajectory toward the sliding surface as well as to establish asymptotic convergence of the tracking error. Consider the Lyapunov function candidate

$$
V_{1}=\frac{1}{2} s_{i}^{2}
$$

raking the derivative of (25) and using (9), (17), one has $\dot{V}_{1}=s_{i}\left(\sum_{j=1}^{r_{j}-1} \alpha_{i j} e_{i}^{\left(r_{i}-f\right)}-f_{i}-\sum_{j=1}^{m} g_{i j} \hat{u}_{j}+y_{d i}^{\left(r_{i}\right)}-d_{t}\right)-s_{i} u_{h i}(26)$ To ensure (26) being less than zero, the hitting control should be sclected as (21). This means that the inequality $\dot{V}_{i}=s_{i} \dot{s}_{i}<0$ is obtained and the hitting control actually achieves a stable FSMC system.

Conceptually, in sliding mode the equivalent control is used when the state trajectory is near $s_{i}=0$, while the hitting control is appended in the case of $s_{i} \neq 0$ [20]. However, the hitting control will generate a very large control force and causes high-frequency unmodelled dynamics [20]. Therefore, we minimize the hitting control in (21) by a fuzzy function in practical implementation [21]. Thus, a fuzzy rule base is of the form

$$
\begin{aligned}
& \text { If } s_{i} \text { is } Z O \text { Then } u_{i} \text { is } u_{i}=\hat{u}_{i} \\
& \text { If } s_{i} \text { is } N Z \text { Then } u_{i} \text { is } u_{i}=\hat{u}_{i}+u_{h i}
\end{aligned}
$$

where $Z O$ and $N Z$ denote zero and nonzero fuzzy sets, respectively, and input variable $s$, is given in (3). The modified control law of the fuzzy controller for (17) is

$$
u_{i}=\frac{\mu_{Z O}\left(s_{i}\right) \cdot \hat{u}_{i}+\frac{\mu_{N Z}}{\left(s_{i}\right)\left[\hat{u}_{i}+\hat{u}_{h i}\right]}}{\mu_{Z O}\left(s_{i}\right)+\mu_{N Z}\left(s_{i}\right)}
$$

where $\mu_{Z \prime}\left(s_{1}\right)$ and $\mu_{N Z}\left(s_{1}\right)$ is the membership functions of fuzzy sets $Z O$ and $N Z$, respectively.

\section{Simulation Results}

We demonstrate the proposed FSMC by the tracking control of a two-link robotic manipulator with 2 degrees of freedom in the rotational angles described by $q=\left(q_{1}, q_{2}\right)^{r}$, as shown in. Fig. 2 . The dynamic equations describing the motion of the robotic system are of the following form [22] 


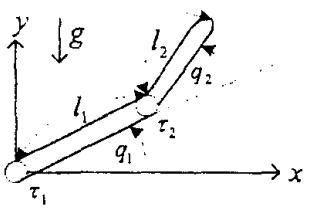

Fig. 2 Model of a two-link robotic manipulator.

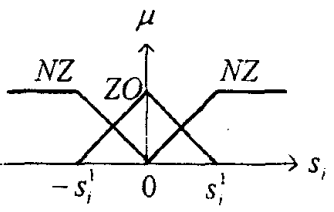

Fig 3 The fuzzy membership functions of $Z O$ and $N Z$.

$$
\boldsymbol{M}(\boldsymbol{q}) \ddot{q}+C(q, \dot{q})+\boldsymbol{h}(q, g)=\boldsymbol{\tau}+\boldsymbol{d}(q, \dot{q}, t)
$$

where $\tau$ is the externally applied torques along the directions of their corresponding generalized coordinates $q$, $M(q)=\left[\begin{array}{cc}\left(m_{1}+m_{2}\right) r_{1}^{2}+m_{2} r_{2}^{2}+2 m_{2} r_{1} r_{2} c_{2}+J_{1} & m_{2} r_{2}^{2}+m_{2} r_{1} r_{2} c_{2} \\ m_{2} r_{2}^{2}+m_{2} r_{1} r_{2} c_{2} & m_{2} r_{2}^{2}+J_{2}\end{array}\right]$ $C(q, \dot{q})=\left[\begin{array}{c}-m_{2} r_{1} r_{2} s_{2} \dot{q}_{1}\left(\dot{q}_{1}+\dot{q}_{2}\right) \\ m_{2} r_{1} r_{2} s_{2} \dot{q}_{2}^{2}\end{array}\right], \quad h(q)=\left[\begin{array}{c}\left.\left(\left(m_{1}+m_{2}\right) l_{1} c_{2}+m_{2} l_{2} c_{12}\right) g\right] \\ \left(m_{2} l_{2} c_{12}\right) g\end{array}\right]$ with $g=9.8 \mathrm{~m} / \mathrm{s}^{2}$ is the gravity constant and $\boldsymbol{d}(\boldsymbol{q}, \dot{\boldsymbol{q}}, t) \in R^{2}$ is friction forces, and external disturbances. In (30), the nominal parameters $m_{l}, m_{2}, J_{l}, J_{2}, r_{1}=0.5 l_{1}$, and $r_{2}=0.5 l_{2}$ are the mass, the moment of inertia, the half-length of link 1 and 2 , and shorthand notations $c_{2}=\cos \left(q_{2}\right), s_{2}=\sin \left(q_{2}\right), c_{12} \equiv \cos \left(q_{1}+q_{2}\right)$, etc. The combined effects of friction and the external torque disturbance are

$$
\begin{aligned}
& d_{1}=2.0 \sin \left(\dot{q}_{1}\right)+2.5 \sin \left(\dot{q}_{2}\right)+0.5 \sin (t) \\
& d_{2}=5.0 \sin \left(\dot{q}_{1}\right)+4.0 \sin \left(\dot{q}_{2}\right)+0.4 \sin (t)
\end{aligned}
$$

In the control experiments described below, the kinematics and inertial parameters of the arm are chosen as $l_{1}=2.04 \mathrm{~m}, l_{2}=1.66 \mathrm{~m}, J_{1}=J_{2}=4.5 \mathrm{~kg} \cdot \mathrm{m}, m_{1}=0.60 \mathrm{~kg}$, $m_{2}=7.02 \mathrm{~kg}$, respectively. The trajectories to be followed are described by two decoupled linear systems from (4), the desired coefficients are specified to be $\alpha_{i 1}=2, \alpha_{i 2}=1$, $i=1,2$. The robot is given the following target joint rotations:

$$
\begin{aligned}
& q_{d 1}=(2.5 \pi / 12) \cdot \sin t \\
& q_{d 2}=(3.75 \pi / 12) \cdot \cos t
\end{aligned}
$$

with the initial states $q_{1}(0)=1.5 \mathrm{rad}, q_{2}(0)=-1.2 \mathrm{rad}$, $\dot{q}_{1}(0)=0 \mathrm{rad} / \mathrm{sec}$ and $\dot{q}_{2}(0)=0 \mathrm{rad} / \mathrm{sec}$.

In $(20)$ and $(21)$, the design parameters are given by $\rho_{i}=1.2, K_{i}=1, D_{i}=5, i=1,2$. The membership functions of states $q_{1}, q_{2}, \dot{q}_{1}$, and $\dot{q}_{2}$ (represented by generic variable $x_{i}$ ) for the qualitative statements ( $N=5^{4}=625$ regular rule partitions) are defined as
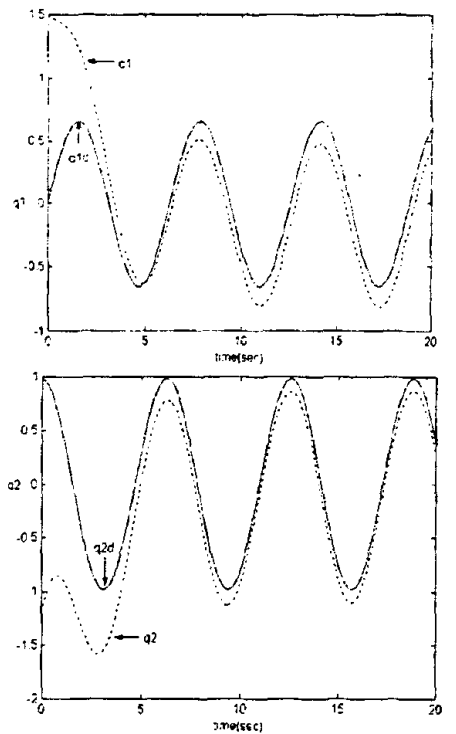

Fig. 4 The tracking curves of $q_{1}, q_{d 1}$ and $q_{2}, q_{d 2}$.
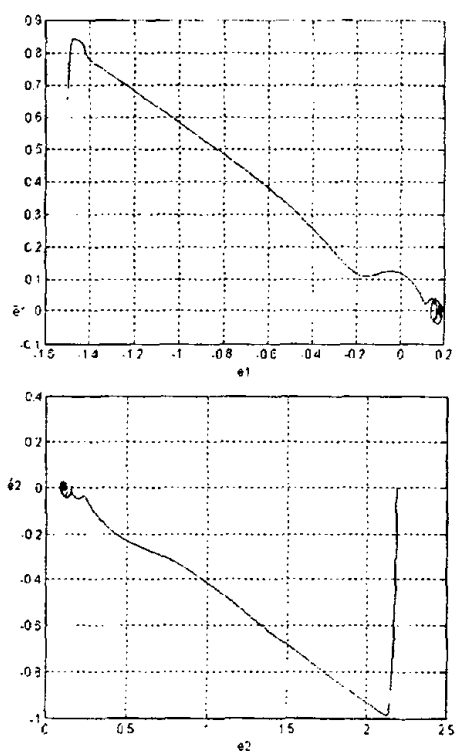

Fig. 5 The state trajectorics on the phase plane.

$\{N B, N S, Z E, P B, P S\}$

where

$N B: 1 /\left[1+\exp \left(2.5\left(x_{i}-0.4\right)\right)\right] \quad, \quad N S: \exp \left(-0.5\left(x_{i}+0.2\right)^{2}\right)$, $P B: 1 /\left[1+\exp \left(-2.5\left(x_{i}-0.4\right)\right)\right], P S: \exp \left(-0.5\left(x_{i}-0.2\right)^{2}\right)$, $Z E: \exp \left(-0.5 x_{i}^{2}\right)$. In (27) and (28), The membership functions of $s_{i}$ for the fuzzy sets $Z O$ and $N Z$ are given in triangle function, as shown in Fig. 3. It has a property that, for all $s_{i}, \mu_{Z O}\left(s_{i}\right)+\mu_{N Z}\left(s_{i}\right)=1$. When holding the 
condition $\left|s_{i}\right| \geq s_{i}^{1}$ with $s_{i}^{1}=0.3$, it can be seen that the control law is the same as the proposed FSMC. However, the amount of hitting control in region $\left|s_{i}\right|<s_{i}^{1}$ is dominated by the grade of the membership function of $N Z$, that is, the hitting control could be attenuated by the gradc of $N Z$.

The tracking curves and the state trajectories of the phase plane for $q /(t)$ and $q_{2}(t)$ are shown in Fig. 4-5, respectively. The simulation results reveal that the proposed FSMC, encountering the combined effects of friction, parametric uncertainties, unmodeled dynamics and external disturbance, can attenuate the tracking error efficiently. Moreover, without using any a priori linguistic information, our adaptive fuzzy sliding mode controller has successfully executed the trajectory following control of the robot system.

\section{Conclusion}

The goal of this work is the development and implementation of a direct adaptive fuzzy control based SMC for the robust trajectory tracking of MIMO control systems with unknown nonlinear dynamics. This design obtains robustness in the sense that the self-tuning mechanism can automatically adapt the fuzzy controller by using a learning algorithm and the global asymptotic stability of the algorithm is established via Lyapunov stability criterion. The simulation presented in the two-link robotic manipulator control indicates that the proposed approach is capable of achieving a good chattering-free trajectory following performance without the knowledge of plant parameters. Although only the two-link robotic system has been studied in this paper, the proposed control scheme can also be used to address the other class of MIMO nonlinear systems.

\section{Acknowledgement}

The financial support for this research from the National Science Council of Taiwan, R. O. C. under NSC90-2212-E002-216 is gratefully acknowledged.

\section{References}

[1] A. Isidori, Nonlinear Control Systems: An Introduction. New York: Springer, 1989.

[2] S. N Singh, "Decoupling of invertible nonlinear systems with state feedback and precompensation," IEEE Trans. Automat. Control, vol. 25, no. 6, pp. $1237-1239,1980$

[3] S. W. Kim and J. J. Lee, "Design of a fuzzy controller with fuzzy sliding surface," Fuzzy Sets and Systems, vol. 71, no. 3, pp. 359-367, 1995.

[4] R. Palm, "Sliding mode fuzzy control," lst IEEE Conf. on Fuzzy Systems, San Diego, USA, March 1992, pp. 519-526.

[5] R. Palm, "Robust control by fuzzy sliding mode," Automatica, vol. 30, no. 9, pp. 1429-1437, 1994.
[6] B. Yoo, "Adaptive fuzzy sliding mode control of nonlinear system," IEEE Trans. Fuzzy Systems, vol. 6, no. 2, pp. 315-321, 1998.

[7] S. B. Choi and J. S Kim, "A fuzzy-sliding mode controller for robust tracking of robotic manipulators," Mechatronics, vol. 2, no. 2, pp. 199 . 216, 1997.

[8] Y. S. Lu and J. S. Chen,"A self-organizing fuzzy sliding-mode controller design for a class of nonlinear servo systems," IEEE Trans. Ind. Electron., vol. 41 , no. 5 , pp. 492-502, 1994

[9] M. Sugeno and G. T. Kang, "Fuzzy modeling and control of multiplayer incinerator," Fuzzy Sets and Systems, vol. 18, no. 3, pp. 329-346, 1986.

[10] M. Sugeno and G. T. Kang, "Fuzzy identification of fuzzy model," Fuzzy Sets and Systems, vol. 28, no. 1, pp. 15-33, 1988.

[11] T. Takagi and M. Sugeno, "Fuzzy identification of systems and its application to modeling and control," IEEE Trans. Syst. Man, Cybern., vol. 15, no. 1, pp. 116-132, 1985.

[12] K. Tanaka and M. Sugeno, "Stability analysis and design of fuzzy control systems," Fuzzy Sets and Systems, vol. 45, no. 2, pp. 135-156, 1992.

[13] L. X. Wang, A course in fuzzy systems and control. New Jersey: Prentice Hall, 1997.

[14] L. X. Wang, "Stable adaptive fuzzy control of nonlinear systems," IEEE Trans. Fuzzy Systems, vol. 1, no. 2, pp. 146-155, 1993.

[15] J. L. Castro "Fuzzy logic controllers are universal approximators," IEEE Trans. Syst. Man, Cybern., vol. 25, no.4, pp. 629-635, 1995.

[16] C. T. Lin, and C. S. G. Lee, 'Neural-network-based fuzzy logic control and decision system', IEEE Trans. Computers, vol. 40, no. 12, pp. 1320-1336, 1991.

[17] W. S. Lin and C. S. Chen, "Adaptive fuzzy sliding mode controller design for nonlinear MIMO systems," in Proc. IEEE Systems, Man, and Cybernetics Conference, October 2001, Tucson, USA.

[18] J. J. E. Slotine and J. A. Coetsee, "Adaptive sliding controller synthesis for non-linear systems," Int. $J$. Control, vol. 43, no. 6, pp. 1631-1651, 1986.

[19] R. A. Hom and C. R. Johnson, Matrix Analysis. Cambridge, U. K: Cambridge University Press, 1985.

[20] V. I. Utkin, "Variable structure systems with sliding modes," IEEE Trans. Automat. Control, vol. 22, no.2, pp. 212-22, 1977.

[21] J. Y. Chen: 'Rule regulation of fuzzy sliding mode controller design: direct adaptive approach', Fuzzy Sets and Systems, vol.120, no. 1, pp. 159-168, 2001.

[22] T. P. Leung, Q. J. Zhou and C. Y. Su, "An adaptive variable structure model following control design for robot manipulators," IEEE Trans. Automatic Control, vol. 36, no. 3, pp. 347-353, 199 I. 